LA CULTURA ORGANIZACIONAL Y LA SATISFACCIÓN LABORAL EN LA DELEGACIÓN FEDERAL DE LA SECRETARÍA DE EDUCACIÓN PÚBLICA (SEP) EN EL ESTADO DE TABASCO

\title{
LA CULTURA ORGANIZACIONAL Y LA SATISFACCIÓN LABORAL EN LA DELEGACIÓN FEDERAL DE LA SECRETARÍA DE EDUCACIÓN PÚBLICA (SEP) EN EL ESTADO DE TABASCO
}

Trinidad Pérez Jesahel * García Rodríguez José Félix **

* Estudiante de la Licenciatura en Administración. Universidad Juárez Autónoma de Tabasco (UJAT). División Académica de Ciencias Económico Administrativas (DACEA).

** Doctor en Finanzas Públicas. Profesor Investigador de la Universidad Juárez Autónoma de Tabasco, División Académica de Ciencias Económico Administrativas.

Dirección para recibir correspondencia: jetripe@gmail.com 
LA CULTURA ORGANIZACIONAL Y LA SATISFACCIÓN LABORAL EN LA DELEGACIÓN

FEDERAL DE LA SECRETARÍA DE EDUCACIÓN PÚBLICA (SEP) EN EL ESTADO DE TABASCO

\section{RESUMEN}

OBJETIVO: Conocer la percepción de cultura organizacional en la Delegación Federal de la SEP en el Estado de Tabasco y la satisfacción laboral de sus trabajadores.

MATERIAL Y MÉTODO: Se realizó un estudio transversal descriptivo en los trabajadores de la Delegación Federal de la SEP en el Estado Tabasco. La investigación tuvo un enfoque cuantitativo, mediante un censo de 30 colaboradores activos al momento del estudio. El instrumento para la recopilación de la información es un cuestionario estructurado y clasificado en tres bloques.

RESULTADOS: Relacionados con los constructos de los cuatro tipos de culturas: de clan, de adhocracia, de mercado y jerarquizada. Se encontró que el tipo de cultura organizacional predominante en la organización es la de mercado; en relación con la satisfacción laboral, el $45 \%$ del personal encuestado manifestó encontrarse muy satisfecho con su trabajo. El nivel más alto de insatisfacción, es relacionada con el bajo nivel de remuneración y la falta de capacitación

CONCLUSIONES: El éxito de toda organización reside en la capacidad de integrar a los trabajadores en sus proyectos, de tal modo que estos consideren los objetivos de la institución como propios. Las instituciones están empezando a comprender que los empleados no se retienen únicamente mediante el salario, sino también por medio de todos aquellos factores que influyen en el grado de satisfacción, como puede ser el reconocimiento, remuneración, capacitación, relación con su jefe de área, flexibilidad, posibilidad de ascenso.

PALABRAS CLAVE: Cultura organizacional. Satisfacción laboral. Desempeño laboral. Organización. 
LA CULTURA ORGANIZACIONAL Y LA SATISFACCIÓN LABORAL EN LA DELEGACIÓN FEDERAL DE LA SECRETARÍA DE EDUCACIÓN PÚBLICA (SEP) EN EL ESTADO DE TABASCO

En la actualidad, dentro de un ambiente de competitividad y rentabilidad en el que las organizaciones requieren adaptarse y responder de forma efectiva al ambiente externo, la comprensión de la cultura organizacional debe ser estratégica en cualquier tipo de organización, incluyendo las que se centran en la educación superior.

La cultura organizacional es la parte esencial de una organización, ya que ésta, se forma por las personas que integran dicha institución, por lo cual es de suma importancia que todos los integrantes de la organización comprendan su significado y la relevancia que éste tiene a la hora de realizar las diversas tareas laborales dentro de la empresa.

Los estudios de cultura organizacional en las Instituciones de Educación Superior, generalmente han estado asociados a variables como satisfacción, motivación laboral e innovaciones pedagógicas, con la oportunidad de abordar el tema desde la perspectiva de evaluación del desempeño.

La cultura organizacional parece ser el mecanismo a través del cual las organizaciones fomentan el aprendizaje e influyen en la percepción y participación de la gente en la gestión de conocimiento. De ahí la importancia de que las organizaciones promuevan y construyan valores culturales y establezcan iniciativas para la gestión de conocimiento; y fomenten el uso de sistemas y tecnologías que puedan potencializar el desarrollo de éste (Alavy, 2005 y Delong y Fahey, 2000) citado por Ynzunza (2013, p. 78).

La función educativa en México, ha sido una preocupación permanente y prioritaria para la administración pública federal, por su papel decisivo en el desarrollo integral del país. Desde su creación, en 1921, la Secretaría de Educación Pública ha asumido esa responsabilidad generando la infraestructura necesaria en materia de políticas, servicios y estructuras organizacionales que, luego de décadas de esfuerzo, han permitido obtener una educación pública de alcances nacionales.

El desarrollo del país y el incremento de la población, han significado para la SEP un tenaz proceso de adaptación ante la exigencia de responder sin demora a nuevas necesidades educativas. El Acuerdo Nacional para la Modernización de la Educación Básica, suscrito en 
LA CULTURA ORGANIZACIONAL Y LA SATISFACCIÓN LABORAL EN LA DELEGACIÓN FEDERAL DE LA SECRETARÍA DE EDUCACIÓN PÚBLICA (SEP) EN EL ESTADO DE TABASCO

mayo de 1992, planteó para esta Secretaría tres grandes compromisos: consolidación del federalismo educativo; reformulación de contenidos y materiales educativos; y revaloración de la función magisterial.

La expedición de la Ley General de Educación señaló el nuevo marco de corresponsabilidad entre la Federación y las entidades federativas para la prestación de los servicios de educación básica y normal. La incorporación e integración de nuestro país a la actividad económica y comercial mundiales, exigen de mayores esfuerzos para generar la educación de calidad y alto grado de competitividad para todos los mexicanos. Estos son sólo algunos de los elementos que definen el entorno al cual la Secretaría debe estar incorporada como organización cambiante.

\section{Oficina de Servicios Federales de Apoyo a la Educación en Tabasco (OSFAE)}

Es una Unidad Administrativa de la SEP adscrita orgánicamente a la Coordinación General de Oficinas de Servicios Federales de Apoyo a la Educación, creada a partir de 1992 como una oficina de enlace entre las Autoridades Educativas Federales y Estatales, derivado de los cambios que impulsó el federalismo educativo en el país.

Desde entonces a la fecha, la OSFAE ha jugado un papel muy importante propiciando el sano desarrollo del Sistema Educativo Estatal y el cumplimiento de las Normas Nacionales, realizando todas aquellas funciones que dentro del ámbito de su competencia procuran el buen funcionamiento de la Política Educativa.

La Misión de la OSFAE es, representar a la Secretaría de Educación Pública y a su titular como instancia única ante las autoridades de los estados de la República; coordinar y dar seguimiento a programas y proyectos educativos; así como generar y proporcionar información oportuna y relevante para la toma de decisiones, que mejoren la calidad de los servicios educativos.

La principal problemática que enfrenta la OSFAE, radica en que no se cuenta con una estructura acorde a los perfiles de puestos requeridos para el desempeño de sus funciones, así como las limitaciones presupuestales para la adecuada operación de los servicios. 
LA CULTURA ORGANIZACIONAL Y LA SATISFACCIÓN LABORAL EN LA DELEGACIÓN FEDERAL DE LA SECRETARÍA DE EDUCACIÓN PÚBLICA (SEP) EN EL ESTADO DE TABASCO

Para dar cumplimiento a sus funciones enmarcadas en el Reglamento Interior de la Secretaría de Educación Pública y frente al nuevo horizonte prospectivo y transformacional de la Secretaría de Educación Pública, que se define con claridad en el Programa Nacional de Educación 2012-2018, elaboró su Programa de Trabajo Anual 2013, que sin duda, permitirá medir y cuantificar el grado de avance en el cumplimiento de los objetivos y metas establecidas.

En este sentido, el Programa Anual de Trabajo de la Oficina de Servicios Federales de Apoyo a la Educación en Tabasco, es un instrumento importante de planeación y programación en las tareas a desarrollar por el personal de mandos y operativos de esta Oficina.

El 1 de octubre del año 2013, desaparecen las OSFAES y regresan las Delegaciones Federales de la SEP en los Estados de la República, donde su misión es: Crear condiciones que permitan asegurar el acceso de todas las mexicanas y mexicanos a una educación de calidad, en el nivel y modalidad que la requieran y en el lugar donde la demanden.

\section{MARCO TEÓRICO}

\section{Cultura organizacional}

De acuerdo con Hernández y Sánchez (1988, p. 247), esta idea inició en la década los cincuenta y se popularizó entre los años setenta y ochenta, tiempo en el que las organizaciones empezaron a considerar el factor humano para asegurar sus metas.

El interés por el estudio de la cultura organizacional se introdujo en la teoría de la gestión por aportaciones de diferentes autores como Elliot Jacques, quien establece una de las primeras definiciones de cultura organizacional (Aktouf, 2002, p. 64); Edgar Schein (1992), citado por Zalpa (2002, p.16), quien define cultura organizacional como un patrón de suposiciones básicas compartidas, que el grupo aprende en la medida que da solución a sus problemas de adaptación externa e integración interna, que han funcionado lo suficientemente bien para ser consideradas válidas para enseñarse a los nuevos miembros; y Smircich (1983) citado por Soria (2008, p.5), quien refiere cinco temas en los que convergen la cultura y la organización: administración comparada, cultura corporativa, 
LA CULTURA ORGANIZACIONAL Y LA SATISFACCIÓN LABORAL EN LA DELEGACIÓN FEDERAL DE LA SECRETARÍA DE EDUCACIÓN PÚBLICA (SEP) EN EL ESTADO DE TABASCO

conocimiento organizacional, simbolismo organizacional, y procesos inconscientes en la organización.

Koys y DeCotiis citado por Chiang, Salazar y Núñez (1991), señala que estudiar el clima en las organizaciones ha sido difícil debido a que se trata de un fenómeno complejo y con múltiples niveles; sin embargo, se ha producido un avance considerable en cuanto al concepto del clima.

Para Taguiri y Litwin citado por Vázquez y Guadarrama (2001) lo definen como una cualidad relativamente duradera del medio ambiente interno de una organización que: experimentan sus miembros, influye en su comportamiento, y se puede describir en términos de los valores de una serie particular de características (atributos) de la organización.

Davis (1987), dice que el clima organizacional es el ambiente humano dentro del cual realizan su trabajo los empleados de alguna compañía, menciona que el clima influye en la motivación, en el desempeño y en la satisfacción en el empleo.

La importancia que tiene la cultura organizacional dentro de una empresa es fundamental, ya que ayuda a que el empleado se sienta en un ambiente laboral confiable que lo motive a realizar las actividades que desempeña.

La cultura organizacional refleja los valores y actitudes de los miembros que a su vez lo convierte en sus elementos, su importancia consiste en analizar y evaluar el clima de una organización por tres razones (Brunet, 2002):

- Evaluando las fuentes de conflictos, estrés o de insatisfacción que contribuyan el desarrollo de actitudes negativas frente a la organización.

- Iniciar y sostener un cambio que indique al administrador los elementos específicos sobre los cuales debe dirigir sus intervenciones.

- Seguir el desarrollo de su organización y prever los problemas que puedan surgir.

La cultura organizacional presenta un ciclo constante: se percibe, aprende, transmite y modifica. 
LA CULTURA ORGANIZACIONAL Y LA SATISFACCIÓN LABORAL EN LA DELEGACIÓN FEDERAL DE LA SECRETARÍA DE EDUCACIÓN PÚBLICA (SEP) EN EL ESTADO DE TABASCO

Los componentes de la cultura organizacional como el comportamiento de los individuos y los grupos, la estructura y los procesos organizacionales, hacen que produzca resultados que se observen en el rendimiento organizacional, individual o de grupo. Así, los resultados que se observan en una organización provienen de su tipo de cultura que es resultado de los diferentes aspectos objetivos de la realidad de la organización como la estructura, los procesos y los aspectos psicológicos y el comportamiento de los empleados.

Los elementos y factores que se estudian dentro del clima organizacional son: estructura, motivación, trabajo en equipo, liderazgo, participación del trabajador, toma de decisiones, comunicación, responsabilidad, trabajo significativo y desafiante y conflicto. Dichos elementos son los más representativos para que surjan ideas que posteriormente se cristalicen como innovaciones, pues es necesario que los miembros de la organización sientan, que son parte integral de ésta, que son tomados en cuenta, que no deben temer a opinar y de hecho a criticar los elementos de la organización que no contribuyen a los procesos de cambio, proponiendo nuevas formas de realizar las actividades y/o labores, entre otras actitudes que se fomentan en los individuos, cuando el clima organizacional es propicio para esto (ver figura 1).

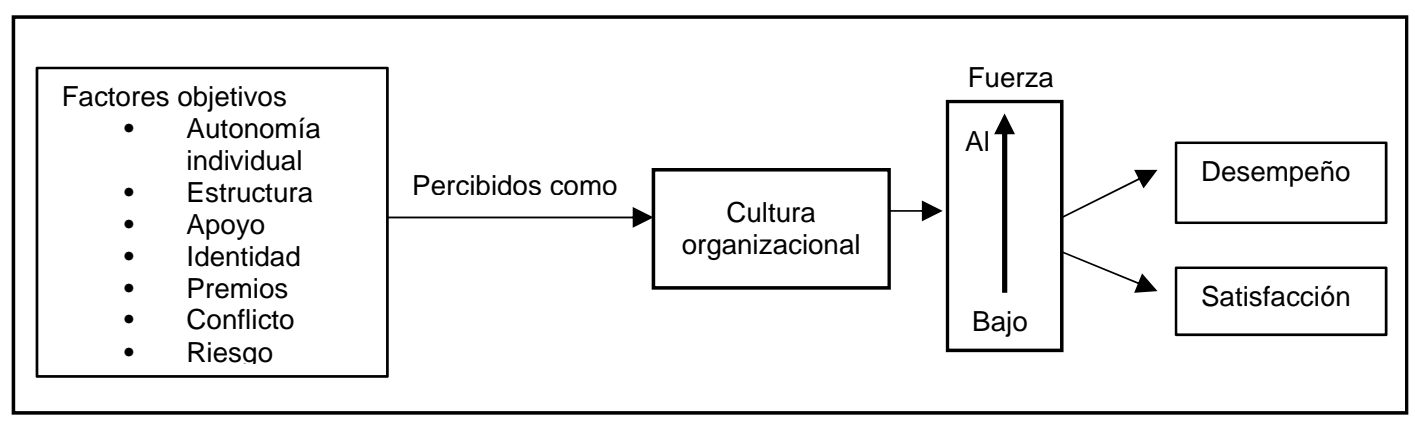

Figura 1. Cómo la cultura organizacional influye en el desempeño y la satisfacción.

Fuente: Comportamiento organizacional: conceptos, controversias y aplicaciones de Stephen P. Robbins (1987).

El clima organizacional constituye cada vez más un factor que refleja las facilidades o dificultades que encuentra el trabajador para aumentar o disminuir su productividad o para encontrar su satisfacción.

Por lo tanto, evaluando el clima laboral lo que se está haciendo es determinar qué tipo de dificultades existen en una organización a nivel de recursos humanos y organizacionales, 
LA CULTURA ORGANIZACIONAL Y LA SATISFACCIÓN LABORAL EN LA DELEGACIÓN FEDERAL DE LA SECRETARÍA DE EDUCACIÓN PÚBLICA (SEP) EN EL ESTADO DE TABASCO

internos o externos que actúan facilitando o dificultando los procesos que conducirán a la productividad de los trabajadores y todo el sistema organizacional.

El primer paso para conocer a una organización es conocer su cultura. Formar parte de una empresa, trabajar en ella, participar en sus actividades y desarrollar una carrera implica asimilar su cultura organizacional o su filosofía corporativa. La forma en que interactúan las personas, las actitudes predominantes, los supuestos subyacentes, las aspiraciones y los asuntos relevantes de las interacciones humanas forman parte de la cultura de la organización (Chiavenato, 2009, p. 123).

Para Chiavenato (2009), la cultura organizacional tiene seis características principales:

1. Regularidad de los comportamientos observados. Las interacciones entre los miembros se caracterizan por un lenguaje común, terminología propia y rituales relativos a las conductas y diferencias.

2. Normas. Pautas de comportamiento, políticas de trabajo, reglamentos y lineamientos sobre la manera de hacer las cosas.

3. Valores dominantes. Son los principios que defiende la organización y que espera que sus miembros compartan, como calidad de los productos, bajo ausentismo y elevada eficiencia.

4. Filosofía. Las políticas que reflejan las creencias sobre el trato que deben recibir los empleados a los clientes.

5. Reglas. Guías establecidas que se refieren al comportamiento dentro de la organización. Los nuevos miembros deben aprenderlas para ser aceptados en el grupo.

6. Clima organizacional. La sensación que transmite el local, la forma en que interactúan las personas, el trato a los clientes y proveedores.

Una vez establecida una cultura, hay prácticas dentro de la organización que la conservan al dar a los integrantes una serie de experiencias semejantes. El proceso de selección, los criterios con que se evalúa el desempeño, los métodos de concesión de premios, las actividades conexas con la capacitación y el desarrollo de carreras, los procedimientos de promoción garantizan que los nuevos empleados encajen en la cultura, premian a quienes la apoyan y castigan (llegando incluso al despido) a quienes se opongan a ella. 
LA CULTURA ORGANIZACIONAL Y LA SATISFACCIÓN LABORAL EN LA DELEGACIÓN FEDERAL DE LA SECRETARÍA DE EDUCACIÓN PÚBLICA (SEP) EN EL ESTADO DE TABASCO

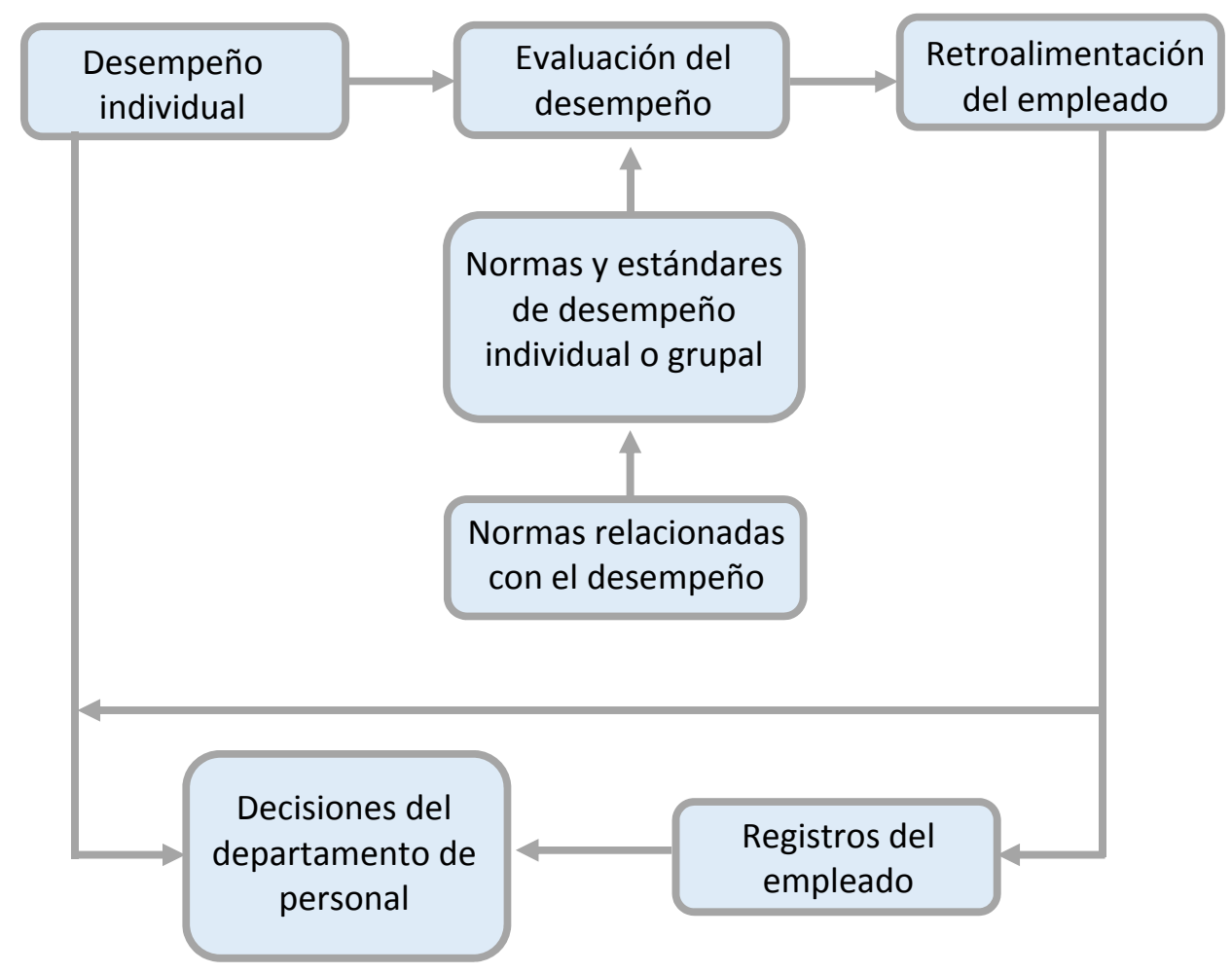

Figura 2. Elementos claves en los sistemas de evaluación del desempeño.

Fuente: Administración de recursos humanos. El capital humano de las empresas de William y Keith (2008).

Schein (1988, p. 64) considera funcional una cultura organizacional que contribuya a la “integración interna" mediante la cohesión de sus miembros o a la "adaptación externa" a los cambios contextuales.

Su acercamiento funcionalista permite comprender lo que la cultura hace para "resolver los problemas básicos de un grupo social".

- La construcción de relaciones externas para la supervivencia y adaptación en el medio que lo rodea.

- La integración de sus procesos internos para afianzar su capacidad de supervivencia y adaptación. 
LA CULTURA ORGANIZACIONAL Y LA SATISFACCIÓN LABORAL EN LA DELEGACIÓN

FEDERAL DE LA SECRETARÍA DE EDUCACIÓN PÚBLICA (SEP) EN EL ESTADO DE TABASCO

\section{Satisfacción laboral}

Schneider (1985), indica algunas razones que explican la gran atención dedicada a la satisfacción laboral: 1) La satisfacción en el trabajo es un resultado importante de la vida organizacional. 2) La satisfacción ha aparecido en diferentes investigaciones como un predictor significativo de conductas disfuncionales importantes, como el absentismo, el cambio de puesto y de organización.

De acuerdo a los hallazgos, investigaciones y conocimientos acumulados (Robbins, 1998) considera que los principales factores que determinan la satisfacción laboral son:

- Reto del trabajo.

- Sistema de recompensas justas.

- Condiciones favorables de trabajo.

- Colegas que brinden apoyo.

Adicionalmente:

- Compatibilidad entre personalidad y puesto de trabajo.

Una definición difundida es la propuesta por Davis y Newstrom (1999), según la cual la satisfacción es concebida como un concepto multidimensional, que incluye un conjunto de sentimientos favorables y desfavorables del individuo frente a su trabajo y que agrupa diversos factores, distinguiendo entre aquellos que están directamente relacionados con la naturaleza de la tarea desempeñada y aquellos relativos al contexto laboral.

En cuanto a la importancia de la satisfacción laboral para las organizaciones, a lo largo de la revisión de la literatura especializada, se observa que, en términos generales, existe una relación positiva entre la satisfacción de los empleados y los resultados de la organización (Ídem, 1999).

Para Robbins (2004) la satisfacción en el trabajo, está determinada por el atractivo de la tarea, las recompensas asociadas, las relaciones con los compañeros de trabajo y las condiciones laborales a las que los empleados están expuestos. Además, afirman que otros factores relacionados con la personalidad del empleado y con las características de la función que desempeña, intervienen de igual manera en la percepción que el empleado tiene de su bienestar y complacencia en lo que hace, así en la medida en la que la 
LA CULTURA ORGANIZACIONAL Y LA SATISFACCIÓN LABORAL EN LA DELEGACIÓN FEDERAL DE LA SECRETARÍA DE EDUCACIÓN PÚBLICA (SEP) EN EL ESTADO DE TABASCO

personalidad concuerde con la vocación elegida, existirá una mayor posibilidad que tener unos trabajadores satisfechos.

Meliá y Peiró (1989) recogen como factores separados la satisfacción con la supervisión y la satisfacción con la participación, además de la satisfacción con el ambiente físico, la satisfacción con las prestaciones y la satisfacción intrínseca.

En la actualidad, el fenómeno de la satisfacción laboral adquiere una vital importancia para el desarrollo de la humanidad. Resulta evidente que es necesario combinar las nuevas tecnologías y el factor humano para lograr la eficacia, eficiencia y efectividad en las organizaciones del siglo XXI.

En la comunidad científica, existe consenso en señalar que la satisfacción laboral es la actitud que asume la persona ante su trabajo y que se refleja en actitudes, sentimientos, estados de ánimo y comportamientos en relación a su actividad laboral.

Si la persona está satisfecha con su trabajo responderá adecuadamente a las exigencias de este; si, por el contrario, está insatisfecha no será capaz de realizar su labor con eficiencia y calidad. Además, la insatisfacción laboral se refleja en todas la esferas de la vida del trabajador (García, 2010).

La satisfacción en el trabajo es importante en cualquier tipo de trabajo, no sólo en términos del bienestar deseable de las personas dondequiera que trabajen, sino también en términos de productividad y calidad. En el caso de las Instituciones Públicas, la variable de satisfacción laboral reviste singular importancia desde el ámbito de la calidad de la gestión de los grupos de trabajo que ellos forman al interior de su institución.

\section{MATERIAL Y MÉTODO}

Se realizó un estudio transeccional descriptivo, aplicado a los trabajadores de la Delegación Federal de la SEP en la ciudad de Villahermosa, Tabasco. La investigación fue cuantitativa debido al tipo de estudio y al tamaño de la muestra que fueron de 30 casos.

El criterio utilizado para la selección de la muestra, fue que los participantes estuvieran trabajando para la delegación y contaran por lo menos con 2 años de experiencia. 
LA CULTURA ORGANIZACIONAL Y LA SATISFACCIÓN LABORAL EN LA DELEGACIÓN FEDERAL DE LA SECRETARÍA DE EDUCACIÓN PÚBLICA (SEP) EN EL ESTADO DE TABASCO

El cuestionario solamente consideró 35 ítems, los cuales fueron evaluados mediante una escala de Likert de 5 puntos. La primera parte del cuestionario considera preguntas sociodemográficas como son: género, edad, antigüedad laboral, experiencia, entre otros, la segunda evalúa los aspectos relacionados con la cultura organizacional y la tercera explora el grado de satisfacción laboral.

Respecto a los constructos que forman la escala de medición, para la cultura organizacional se utilizó la escala OCAI (Organizacional Culture Assessment Instrument) propuesta por Cameron y Quinn (1999). La cual considera 4 perfiles de cultura:

- Cultura de Clan: Tipo de organización familia, donde la organización es un lugar muy amistoso para trabajar y donde las personas comparten mucho entre sí.

- Cultura Adhocracia: También llamada cultura empresarial, que representa el tipo de organizaciones que son altamente sensibles a los cambios acelerados.

- Cultura Jerarquizada: La organización es un lugar estructurado y formalizado para trabajar, los procedimientos gobiernan y dicen a las personas qué hacer.

- Cultura de Mercado: Donde el orden interno está garantizado por las normas y con seis criterios de evaluación: características dominantes, líderes organizacionales, manejo de empleados, unión organizacional, énfasis estratégico y criterio de éxito.

Para la ratificación de la validez de contenido, se procedió a la entrega del instrumento aplicados, para medir el grado de satisfacción con 10 ítems del empleo y la organización, (medido en la escala de Likert de 5 puntos, siendo 1 "muy insatisfecho" y 5 "muy satisfecho") y satisfacción con el trabajo de forma general; las cuales son: remuneración, reconocimiento, capacitación, relación con su jefe de área, flexibilidad, posibilidad de ascenso, condiciones de trabajo, integración en el trabajo, iniciativa y retroalimentación.

Asimismo, se realizó la validez de experto según Hernández, Fernández y Baptista (2014), la cual se refiere al grado en que aparentemente un instrumento mide la variable en cuestión, de acuerdo con "voces calificadas", donde se resumieron las condiciones realizadas por cada ítem.

La confiabilidad se determinó bajo la aplicación de una prueba piloto del instrumento, para lo cual se aplicó el cuestionario al 15\% de la población, ajustándose algunas preguntas con 
LA CULTURA ORGANIZACIONAL Y LA SATISFACCIÓN LABORAL EN LA DELEGACIÓN FEDERAL DE LA SECRETARÍA DE EDUCACIÓN PÚBLICA (SEP) EN EL ESTADO DE TABASCO

base en los comentarios y dudas de los participantes. Para confiabilizar el instrumento se utilizaron técnicas estadísticas y de fiabilidad como el Alfa de Cronbach. El Alfa de Cronbach del cuestionario fue de .947; los valores de confiabilidad por constructo se sitúan de .825 a .920. Los resultados encontrados presentan evidencia de la confiabilidad y validez del instrumento.

En el procedimiento de recolección de datos se les entregó a los trabajadores un cuestionario dentro de un sobre blanco para garantizar el total anonimato. Aproximadamente dos semanas después de esta primera toma se procedió a recoger los sobres cerrados con las encuestas.

Para el tratamiento de los datos, se empleó un análisis descriptivo, que permite obtener los datos básicos de estudio: género, edad, nacionalidad, categoría, unidad de trabajo, funciones, salario, antigüedad, departamento, horas de trabajo y turno, determinando así las diferencias dadas como resultados por dichas variables. Para medir la cultura organizacional, de acuerdo a la escala de medición utilizada, se evaluaron los cuatro perfiles de cultura; así mismo, para medir el nivel de satisfacción laboral, se hizo un comparativo de las medias obtenidas de cada uno de los diez ítems.

\section{RESULTADOS}

Una adecuada cultura organizacional contribuye de manera significativa al avance de la organización, donde la actitud de cada uno de los miembros está orientada al beneficio grupal y no individual. Existen diferencias en cuanto al impacto de la cultura organizacional, al igual que en los niveles de satisfacción y el desempeño laboral; sin embargo, estos conceptos permitirán el mejoramiento en la Delegación Federal SEP, aportando procesos de innovación y sus procedimientos en cada una de sus áreas, así como la comunicación bidireccional entre la dirección y sus colaboradores, el cual fortalecerá a la organización en su desarrollo. Deal y Kennedy (1993), afirman que los valores comunes de la organización forman el núcleo de la cultura, por ello la organización debe trabajar sobre estos valores comunes y proporcionar "héroes modélicos" y "líderes" a quien los demás intentan imitar. Respecto a los datos sociodemográficos, se encontró que el 85\% son del sexo femenino, distribuidos en: directores, jefes de área y secretarias; y el 15\% de la población de sexo masculino, distribuido en jefes de área y auxiliar. El 100\% cuenta con contrato, lo que habla 
LA CULTURA ORGANIZACIONAL Y LA SATISFACCIÓN LABORAL EN LA DELEGACIÓN FEDERAL DE LA SECRETARÍA DE EDUCACIÓN PÚBLICA (SEP) EN EL ESTADO DE TABASCO

de una sensación de seguridad. Según el tipo de contrato la jornada es de 8 horas los 5 días de la semana, a excepción de los días de guardia que les corresponde realizar a los auxiliares.

Con relación a los resultados de los constructos de los cuatro tipos de culturas: de clan, de adhocracia, de mercado y jerarquizada. El tipo de cultura organizacional predominante en la organización es la de mercado (54\%), donde el orden interno está garantizado por las normas y sus criterios de evaluación, es decir organizaciones orientadas a sus resultados, cuya mayor preocupación es realizar un trabajo bien hecho, con personal competitivo orientada a los resultados, donde los líderes son directivos exigentes y competitivos. Seguida por la del Clan (18\%) y la jerarquizada (16\%), el tipo de cultura con menos menciones es la de Adhocracia (12\%) (Ver figura 3).

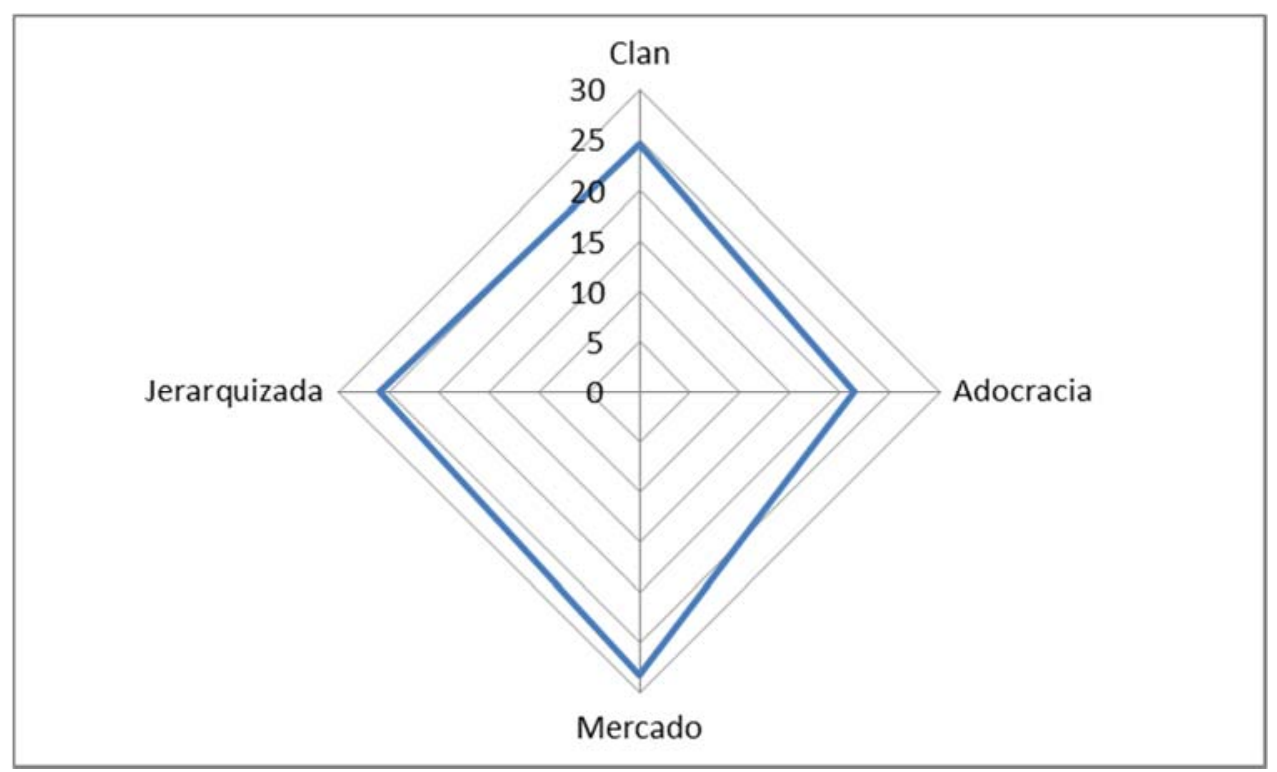

Figura 3. Resultados de la Cultura Organizacional (OCAI).

Fuente: Elaboración propia.

Al evaluar el nivel de satisfacción laboral encontramos que: el 45\% del personal encuestado se encuentra muy satisfecho con su trabajo y el $25 \%$ muy insatisfecho, lo que hace evidente, además de las condiciones sociodemográficas explicadas anteriormente que el $18 \%$ manifiesta su inconformidad con el nivel de ingresos (ver figura 3). 
LA CULTURA ORGANIZACIONAL Y LA SATISFACCIÓN LABORAL EN LA DELEGACIÓN FEDERAL DE LA SECRETARÍA DE EDUCACIÓN PÚBLICA (SEP) EN EL ESTADO DE TABASCO

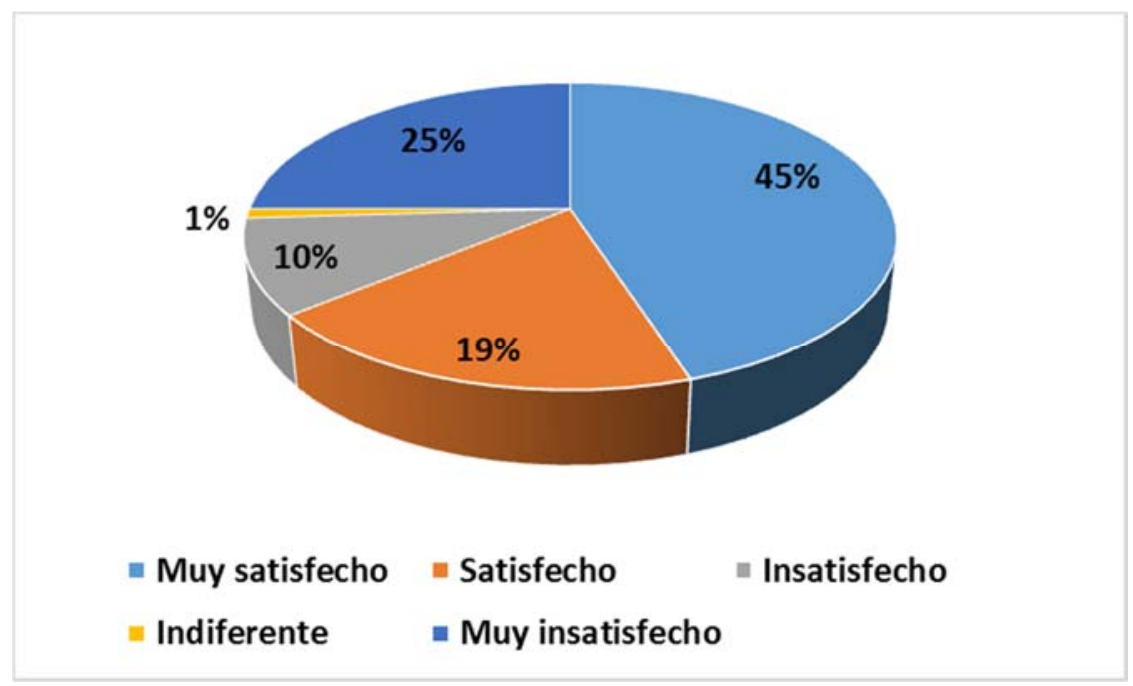

Figura 3. Nivel de satisfacción laboral.

Fuente: Elaboración propia.

Desde el punto de vista de la satisfacción general, la mayoría del personal está satisfecho o muy satisfecho con su trabajo, destacando positivamente la relación con los compañeros y con los superiores, mientras que existe un margen de mejora en los aspectos de desarrollo de la carrera profesional a través de las posibilidades de ascenso y la remuneración.

Conforme a los aspectos vinculados a la actividad laboral que se evaluaron, destacan con una valoración media de 4 puntos, las relaciones con compañeros y la dirección, es decir, la forma en que se les dan las indicaciones los cuales los mantienen muy satisfechos. El siguiente nivel de satisfacción lo constituyen la posibilidad de ascenso, condiciones de trabajo y la autonomía, con un puntaje de 3, lo que indica que se encuentran satisfechos. Esto podría explicarse con el sentido de la pertenencia y de la buena relación que su jefe inmediato logra con ellos haciéndolos sentirse útiles y valorar su trabajo.

El nivel más alto de insatisfacción, que es el 2, es relacionada con el bajo nivel de remuneración y la falta de capacitación (ver figura 4). 


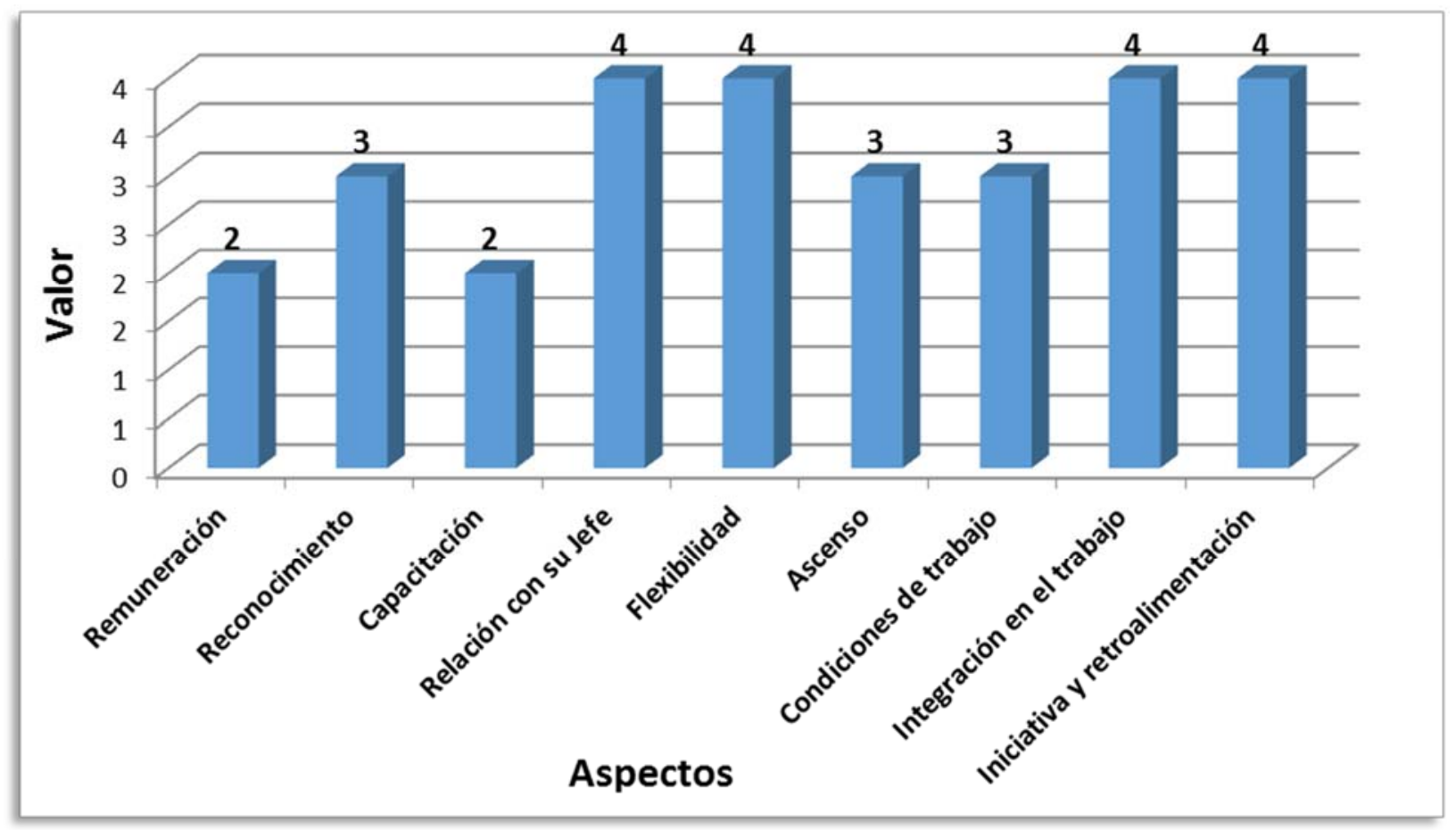

Figura 4. Satisfacción en los distintos aspectos vinculados a la actividad laboral. Fuente: Elaboración propia.

\section{CONCLUSIONES}

La cultura organizacional está relacionada con la gestión de conocimiento, fomenta actitudes positivas hacia éste, generando niveles más altos de satisfacción; debe ser una actividad planeada y sistematizada que permita la identificación, generación y transferencia del conocimiento. Una organización bien direccionada, requiere de una cultura organizacional que aliente a crear y compartir el conocimiento, así como tener buen desempeño laboral, para que los empleados se sientan felices, comprometidos con el trabajo, conectados con los resultados generales y motivados por hacer mayor contribución.

Un elemento fundamental para el éxito de toda organización reside en la capacidad de integrar a los trabajadores en sus proyectos, de tal modo que estos consideren los objetivos de la institución como propios. Las instituciones están comenzando a comprender que los empleados no se retienen únicamente mediante el salario, sino también por medio de todos aquellos factores que influyen en el grado de satisfacción, como puede ser el reconocimiento, remuneración, capacitación, relación con su jefe de área, flexibilidad, posibilidad de ascenso entre otros. 
LA CULTURA ORGANIZACIONAL Y LA SATISFACCIÓN LABORAL EN LA DELEGACIÓN FEDERAL DE LA SECRETARÍA DE EDUCACIÓN PÚBLICA (SEP) EN EL ESTADO DE TABASCO

Las diferencias entre las personas basadas en los aspectos objetivos y/o subjetivos, tienen diferentes consecuencias que, siguiendo a Gallego Águeda y Casanueva Roccha (2010), se pueden resumir en tres:

a) Las personas tienen diferentes respuestas ante un mismo estímulo. Es decir, una mejora salarial no tiene por qué suponer un aumento lineal de la satisfacción laboral o el compromiso organizativo de todos los empleados a los que se les ha aplicado dicho incremento.

b) Las personas no tienen un comportamiento estable a lo largo del tiempo. Es decir, siguiendo el ejemplo anterior, para una persona determinada, la subida salarial realizada en un momento concreto de su carrera profesional, puede tener un alto componente motivador y, en cambio, en otro momento, donde, por ejemplo cuando sus circunstancias personales, sociales o familiares hayan cambiado, el mismo incremento tiene una limitación motivadora neutra. La evolución de un individuo y sus circunstancias, esto es, el cambio, están detrás de este fenómeno.

c) Las personas tiene comportamientos diferentes dependiendo de la dimensión espacial donde se encuentren. Es decir, dependiendo del lugar (físico o figurado) en el que se encuentra una persona, su respuesta a un mismo estímulo puede variar. No nos comportamos igual dentro que fuera del trabajo, con un grupo de personas que con otro, cuando asumimos un rol que otro.

Como parte de la cultura organizacional, es necesario que en la Delegación Federal de la SEP se cuente con un sistema formal de evaluación permanente, que le permita la identificación del conocimiento nuevo, para fomentar actitudes positivas, la integración del personal ante estas y la auto evaluación. 
LA CULTURA ORGANIZACIONAL Y LA SATISFACCIÓN LABORAL EN LA DELEGACIÓN

FEDERAL DE LA SECRETARÍA DE EDUCACIÓN PÚBLICA (SEP) EN EL ESTADO DE TABASCO

\section{REFERENCIAS}

Aktouf, O. (2002). El Simbolismo y la Cultura Organizacional, de los abusos conceptuales a las elecciones de campo. Revista AD-MINISTER. Universidad EAFIT. No. 1. 6393.

Brunet, L. (2002). El clima de trabajo en las organizaciones (5ta reimpresión). México: Trillas.

Cameron \& Quinn. (1999). Diagnosing and changing organizational culture based on the competing values framework EUA: Addison-Wesley Publishing Company, Inc.

Chiang, M., Salazar, M. \& Núñez, A. (2008). Clima organizacional y satisfacción laboral con los resultados, en grupos de docentes de instituciones de educación superior. ICADE.

Chiavenato, I. (2009). Comportamiento organizacional. La dinámica del éxito en las organizaciones. (2da. Ed.). México: McGraw Hill/Interamericana Editores S.A. de C.V.

Davis, J., y Newstrom. (1999). Comportamiento humano en el trabajo (10ª ed.). México: McGraw Hill.

Davis, K. (1987). Comportamiento humano en el trabajo. México: McGraw Hill.

Deal, T. \& Kennedy, A. (1985). Cultura corporativa. México: Fondo Educativo Interamericano.

Gallego Águeda, M.A.; Casanueva Rocha, C. (2010). Dirección y organización de empresas turísticas. Madrid: Ediciones Pirámide.

García, D. (2010). Satisfacción laboral. Una aproximación teórica, en contribuciones de las ciencias sociales. Recuperado en junio de 2010, de www.eumed.net/rev/cccss/09/dgv.htm

Hernández, E. y Sánchez, A. (1998). Aproximaciones para un nuevo modelo de gerencia. Revista Gestión y estrategia. No. 14, enero-junio. 
LA CULTURA ORGANIZACIONAL Y LA SATISFACCIÓN LABORAL EN LA DELEGACIÓN FEDERAL DE LA SECRETARÍA DE EDUCACIÓN PÚBLICA (SEP) EN EL ESTADO DE TABASCO

Hernández, R., Fernández, C., y Baptista, M. (2014). Metodología de la investigación. (6ta. Ed.). México: Mc Graw Hill.

Meliá, J. y Peiró, J. (1989). La medida de la satisfacción laboral en contextos organizacionales. Revista Psicologemas, Vol. 5.

Robbins, S. (1987). Comportamiento Organizacional: Conceptos, controversias y aplicaciones. (3ra ed.). México: Prentice- Hall Hispanoamericana, S.A.

Robbins, S. (1998). Comportamiento organizacional. México: Prentice Hall.

Robbins, S. (2004). Comportamiento organizacional. (10ª ed.). México: Pearson.

Schein, E. (1988). La cultura empresarial y el liderazgo. Barcelona: Plaza \& Janés.

Schneider, B. (1985). Organizational behavior. Annual review psychology, (36).

Soria, R. (2008). La cultura como eje central de una red conceptual comprensiva para el estudio de las organizaciones. Ponencia presentada en VI Congreso Internacional de Análisis Organizacional. Nayarit, México.

Vázquez, R., y Guadarrama, J. (enero, 2001). El clima organizacional en una institución tecnológica de educación superior. Revista internacional de investigación educativa, 3(5), 105-131.

Werther, W., y Keith, D. (2008). Administración de recursos humanos. El capital humano de las empresas (6ta ed.). México: McGraw Hill.

Ynzunza, B., Izar, J., y Ávila, R. (mayo, 2013). Cultura organizacional, gestión de conocimiento y desempeño laboral. Revista Hitos de Ciencias Económico Administrativas, 19(54), 77-86.

Zalpa, G. (2002). La cultura en las organizaciones empresariales: estudios sobre las culturas contemporáneas. Época II, VIII (15) 9-33. 